\title{
Efficacy and safety of ralinepag, a novel oral IP agonist, in PAH patients on mono or dual background therapy: results from a phase 2 randomised, parallel group, placebo-controlled trial
}

\author{
Fernando Torres ${ }^{1}$, Harrison Farber ${ }^{2}$, Arsen Ristic $^{3}$, Vallerie McLaughlin ${ }^{4}$, \\ John Adams ${ }^{5}$, Jinkun Zhang ${ }^{5}$, Preston Klassen ${ }^{5}$, William Shanahan ${ }^{6}$, \\ John Grundy ${ }^{5}$, Ines Hoffmann ${ }^{5}$, Christopher Cabell ${ }^{5}$, Pilar Escribano Subías (1) ${ }^{7}$, \\ Namita Sood ${ }^{8}$, Anne Keogh ${ }^{9}$, Gwyn D'Souza ${ }^{5}$ and Lewis Rubin ${ }^{10}$
}

@ERSpublications

In this 22-week randomised, placebo-controlled phase 2 study of PAH patients on single or dual oral background therapy, ralinepag, an oral IP receptor agonist, significantly reduced PVR. http://bit.ly/ 2XHSccO

Cite this article as: Torres F, Farber H, Ristic A, et al. Efficacy and safety of ralinepag, a novel oral IP agonist, in PAH patients on mono or dual background therapy: results from a phase 2 randomised, parallel group, placebo-controlled trial. Eur Respir J 2019; 54: 1901030 [https://doi.org/10.1183/13993003.010302019].

\section{ABSTRACT}

Purpose: This phase 2 study was designed to assess the efficacy, safety and tolerability of immediaterelease orally administered ralinepag, a selective, non-prostanoid prostacyclin receptor agonist with a 24-h terminal half-life, compared to placebo in adult patients with symptomatic pulmonary arterial hypertension (PAH).

Methods: 61 PAH patients who were receiving standard care, including mono or dual PAH-targeted background therapy were randomised $2: 1$ to ralinepag $(n=40)$ or placebo $(n=21)$. The starting dose of ralinepag was $10 \mu \mathrm{g}$ twice daily. Dosage was then up-titrated as tolerated over the course of the 9-week dose-titration period, to a maximum total daily dose of $600 \mu \mathrm{g}$ (300 $\mu \mathrm{g}$ twice daily). The primary efficacy end-point was the absolute change in pulmonary vascular resistance (PVR) from baseline to week 22. Additional end-points included percentage change in PVR from baseline, other haemodynamic parameters, 6-min walk distance (6MWD) and safety and tolerability.

Results: Ralinepag significantly decreased PVR by 163.9 dyn $\cdot \mathrm{s} \cdot \mathrm{cm}^{-5}$ compared to an increase of 0.7 dyn $\cdot \mathrm{s} \cdot \mathrm{cm}^{-5}$ with placebo $(\mathrm{p}=0.02)$; the least-squares mean change from baseline PVR was $-29.8 \%$ compared with placebo $(\mathrm{p}=0.03)$. 6MWD increased from baseline by $36.2 \mathrm{~m}$ with ralinepag and $29.4 \mathrm{~m}$ with placebo $(\mathrm{p}=0.90)$. Serious adverse events occurred in $10 \%$ of ralinepag patients and $29 \%$ of placebo patients. Study discontinuations occurred in $13 \%$ of ralinepag patients and $10 \%$ of placebo patients. Summary: Ralinepag reduced PVR compared with placebo in PAH patients on mono (41\%) or dual combination (59\%) background therapy.

This study is registered at clinicaltrials.gov with identifier NCT02279160. The study protocol and statistical analysis plan are available on the clinicaltrials.gov website. The database for this clinical trial is the sole property of the sponsor, which has sole authority for the provision of access. 


\section{Introduction}

Pulmonary arterial hypertension (PAH) is characterised by vascular remodelling of the small pulmonary arteries that results in elevated pulmonary vascular resistance (PVR) and right ventricular failure. PAH is thought to be initiated by dysfunction of several key mechanisms influencing vasomotor control, proliferation, inflammation and in situ thrombosis. The vasculopathy is associated with excessive production of growth factors, pro-inflammatory mediators and vasoconstrictors, and a diminished production of potent vasodilators and inhibitors of platelet aggregation $[1,2]$.

Prostacyclin is produced in vascular endothelial cells and normally contributes to vasodilation, inhibition of smooth muscle cell proliferation, inhibition of platelet aggregation and inhibition of inflammation [3, 4]. The functions of prostacyclin in the pulmonary circulation are mediated primarily by a specific cell-surface receptor, the prostacyclin receptor (IP), which is expressed on platelets, smooth muscle and endothelial cells and belongs to the G-protein coupled receptor class. The binding of prostacyclin to the receptor triggers the activation of the G-protein and increases intracellular cAMP, which activates downstream signalling to produce the vasoregulatory effects. Activation of the IP receptor, either through the use of prostacyclin analogues or IP receptor activating drugs, is a means of pharmacologically targeting the diminished prostacyclin synthesis that is characteristic of PAH.

Continuous intravenous infusion of prostacyclin (epoprostenol) was the first PAH-targeted treatment to receive regulatory approval and remains the preferred treatment for those who are severely impaired [5]. However, the clinical use of epoprostenol is challenging due to its poor bioavailability and instability in blood, requiring complex and life-changing drug delivery systems. Accordingly, one of the most important priorities in $\mathrm{PAH}$ therapeutic research, particularly for patients with less-severe disease, has been identification of more chemically stable and bioavailable molecules that specifically target the IP receptor.

Currently approved oral prostacyclin therapies (selexipag and treprostinil) have short half-lives (6.5-13 h for active metabolite of selexipag; $4.5 \mathrm{~h}$ for treprostinil), leading to relatively large fluctuations between peak and trough plasma concentrations. These fluctuations stand in contrast to the continuous therapeutic plasma levels and 24-h IP receptor engagement achieved with continuous i.v. infusion of epoprostenol.

Ralinepag is a next-generation, orally available, non-prostanoid, selective and potent IP agonist which has been studied in human pulmonary tissues [6]. In vitro studies indicate that ralinepag has high binding affinity and selectivity at the human IP receptor [7]. Two earlier phase 1 studies demonstrated a favourable safety and tolerability profile for single (SAD) and multiple ascending doses (MAD) of immediate-release, orally administered ralinepag in healthy volunteers (Arena Pharmaceuticals, San Diego, CA, USA; data on file). Pharmacokinetic data from the SAD/MAD study suggests that the immediate-release formulation of ralinepag yields an effective half-life suitable for twice-daily dosing. The present study was designed to investigate the efficacy, safety and tolerability of twice-daily, orally administered ralinepag immediate-release capsules in adult patients with symptomatic PAH.

\section{Methods \\ Patients and study design}

This was a phase 2, multicentre, international, double-blind, placebo-controlled, randomised trial of 22 weeks' duration. Written informed consent was obtained from all study subjects, and protocols were approved by the institutional review board at each participating study site. Data collection and management were conducted by the study sponsor consistent with a predetermined statistical analysis plan.

The study population included male and female patients between the ages of 18 and 75 years diagnosed with symptomatic PAH of idiopathic or heritable origin, induced by drugs and toxins or associated with connective tissue disease, HIV infection or surgically repaired congenital systemic-pulmonary shunt. Patients were required to be on a stable dose of an oral disease-specific PAH therapy, with either an

Affiliations: ${ }^{1}$ Pulmonary/Critical Care, UT Southwestern Medical Center, William P. Clements Jr University Hospital, Dallas, TX, USA. ${ }^{2}$ Pulmonary and Critical Care, Boston Medical Center, Boston University, Boston, MA, USA. ${ }^{3}$ Dept of Cardiology, Clinical Center of Serbia and Belgrade University School of Medicine, Belgrade, Serbia. ${ }^{4}$ Cardiovascular Medicine, University of Michigan, Ann Arbor, MI, USA. ${ }^{5}$ Arena Pharmaceuticals, San Diego, CA, USA. ${ }^{6}$ Hessian Pharmaceuticals, Hayward, CA, USA. ${ }^{7}$ Dept of Cardiology, Hospital Universitario 12 de Octubre, Complutense University, Madrid, Spain. ${ }^{8}$ University of Texas, Houston, TX, USA. ${ }^{9}$ Heart Transplant, St Vincent's Hospital, Sydney, Australia. ${ }^{10}$ Division of Pulmonary and Critical Care Medicine, University of California, San Diego, CA, USA.

Correspondence: Lewis J. Rubin, Division of Pulmonary and Critical Care Medicine, 9300 Campus Point Drive, M/C7372, University of California, San Diego, La Jolla, CA 92037-1300, USA. E-mail: ljrubinducsd.edu 
endothelin receptor antagonist (ERA) and/or a phosphodiesterase type 5 (PDE5) inhibitor or soluble guanylate cyclase stimulator, for $\geqslant 3$ months and had to remain on stable doses for the duration of the study. Eligible patients demonstrated World Health Organization (WHO)/New York Heart Association (NYHA) functional class II-IV symptoms and a 6-min walk distance (6MWD) of $\geqslant 100 \mathrm{~m}$ and $\leqslant 500 \mathrm{~m}$. The study was conducted in accordance with the ethical principles originating from the Declaration of Helsinki and its amendments, consistent with good clinical practices and local regulatory requirements.

\section{Study procedures}

Eligible patients were randomised 2:1 to ralinepag or placebo. Randomisation was stratified by baseline WHO/NYHA functional class (II versus III or IV). All patients were instructed to take their assigned study drug with food.

Optimal dosing of IP agonists in PAH patients requires titration on an individualised basis up to a maximum tolerated dose in order to mitigate potentially severe side-effects (e.g. nausea, headache, flushing, lightheadedness). The present study consisted of a dose-titration period followed by a maintenance period. The dose-titration period occurred during the first 9 weeks of the study and was followed by a 13-week maintenance period. A follow-up visit was to occur $\sim 3$ weeks after the end of the maintenance period (week 25) (figure 1). Over the course of the 9-week dose-titration period, dosages were up-titrated weekly according to each patient's tolerance, with a maximum daily dose of $600 \mu \mathrm{g}$ (300 $\mu \mathrm{g}$ twice daily). The titration schedule was to begin relatively slowly with $10-\mu \mathrm{g} \cdot \mathrm{day}^{-1}$ increases ending with $200-\mu \mathrm{g} \cdot \mathrm{day}^{-1}$ increases during the last 2 weeks of the titration period (titration schedule total daily dose $20 \mu \mathrm{g}, 30 \mu \mathrm{g}, 40 \mu \mathrm{g}, 60 \mu \mathrm{g}, 80 \mu \mathrm{g}, 120 \mu \mathrm{g}, 160 \mu \mathrm{g}, 200 \mu \mathrm{g}, 400 \mu \mathrm{g}, 600 \mu \mathrm{g})$. Dose reduction was permitted based on tolerability; however, the final dose attained during the titration period was required to be maintained during the 13 -week treatment period prior to evaluation at week 22. Patients were instructed to continue the same dose and regimen of disease-specific PAH medications for the duration of the study.

During the study, the following clinical assessments of efficacy were performed: haemodynamic parameters by right heart catheterisation (RHC), 6MWD, assessment of clinical worsening, N-terminal prohormone of B-type natriuretic peptide (NT-proBNP) levels and WHO/NYHA functional class. Safety was assessed by evaluating adverse events, clinical safety laboratory tests (haematology, serum chemistry, urinalysis), vital signs, 12-lead ECGs and physical examinations. Samples for measurement of NT-proBNP were frozen, batched and sent to a central independent laboratory facility for analysis.

RHC was performed prior to study day 1 of the dose-titration period and at the end of the maintenance period (week 22), $\sim 4 \mathrm{~h}$ after the last dose of study drug. The 6MWD was conducted according to the modified guidelines issued by the American Thoracic Society without specific attention to timing after the prior dose of study drug. Investigators evaluated each patient for indications of clinical worsening throughout the study, based on the predefined criteria of death occurring $\leqslant 14$ days after study drug

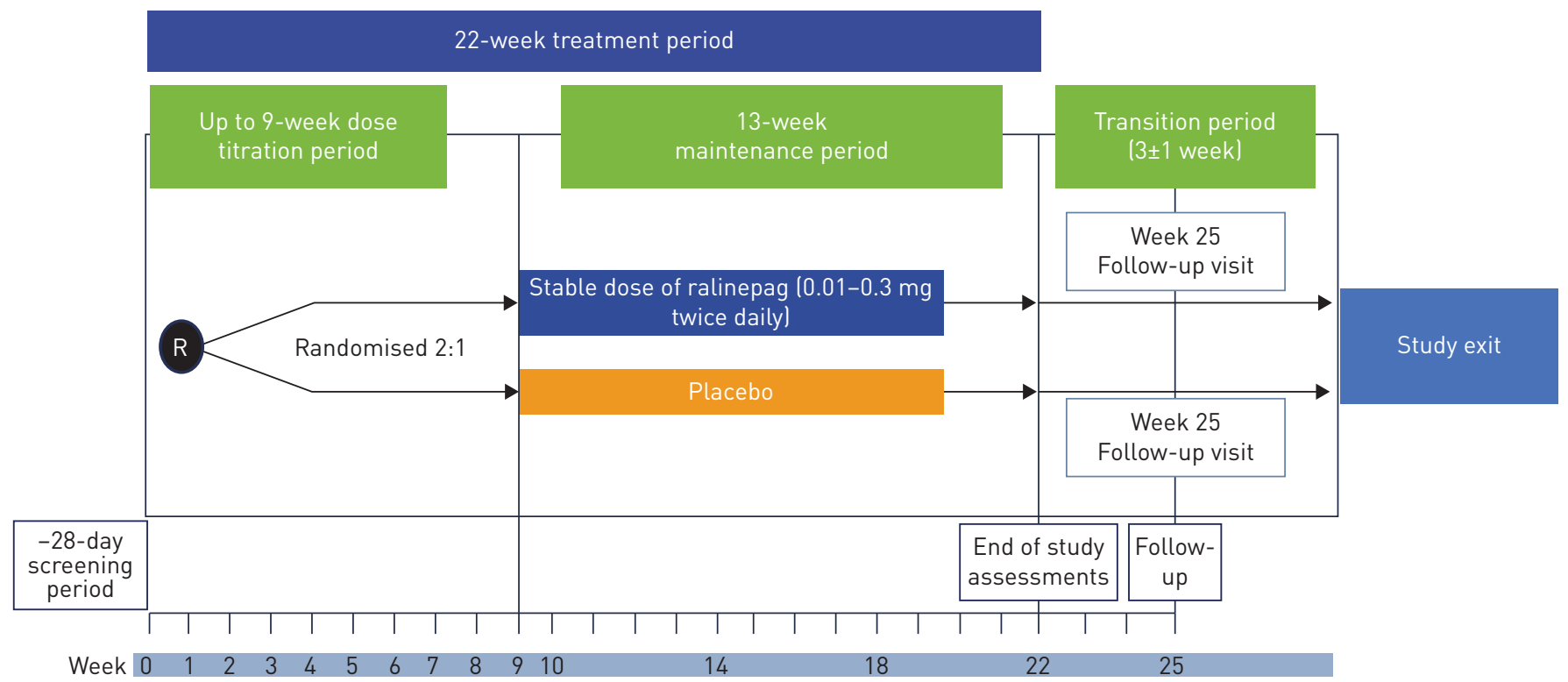


discontinuation, hospitalisation for heart-lung or lung transplantion or atrial septostomy, addition or change in dose of PAH-specific medications, or the combined occurrence of $\geqslant 20 \%$ decrease in $6 \mathrm{MWD}$ from baseline with worsening in WHO/NYHA functional class and worsening of signs of right heart failure that did not respond to optimised oral diuretic therapy.

Details related to any serious adverse events were collected for up to 30 days after the last dose of randomised study medication for patients that withdrew from the study or discontinued randomised, blinded study treatment.

\section{Study end-points}

The primary efficacy end-point was absolute change in PVR from baseline to week 22 with ralinepag compared with placebo. Secondary end-points included percentage change from baseline in PVR, 6MWD, time to clinical worsening, safety and tolerability. Exploratory end-points included changes in other haemodynamic parameters, changes in NT-proBNP and change in WHO/NYHA functional class.

Pharmacokinetic assessments were conducted from blood samples collected and processed for determination of ralinepag plasma concentrations during both the dose-titration and maintenance periods. Blood samples $(\sim 3 \mathrm{~mL})$ were collected pre-dose and at $4 \mathrm{~h}$ post-dose at each study visit where dose escalation was planned during the dose-titration period and during each study visit within the maintenance treatment period.

\section{Statistical analysis}

The sample size estimation of 60 patients was based on the assumption of a between-treatment group difference of $350 \mathrm{dyn} \cdot \mathrm{s} \cdot \mathrm{cm}^{-5}$ mean change in PVR from baseline to week 22 . At this sample size, the study would be powered at $90 \%$ to detect a between-treatment group difference (assuming a 2:1 randomisation ratio) based on a two-sided t-test at the $5 \%$ significance level.

For PVR, assessments measured at week 22 or early withdrawal were included in the analysis.

Missing data at week 22 was imputed using a multiple imputation method. Lastly, to further assess the impact of missing data on the primary analyses, analyses without data imputation using the completers population was performed and compared with additional sensitivity analyses. Baseline assessments were not carried forward.

An analysis of covariance (ANCOVA) model with baseline PVR as a covariate and factors for treatment, baseline WHO/NYHA functional class (II versus III or IV) and baseline background PAH therapy (with ERA versus without ERA) was used to assess the effect of ralinepag versus placebo on change in PVR from baseline. Least-squares means for the treatments and the treatment effect and the least-squares mean difference between treatments was calculated with 95\% confidence intervals and a two-sided p-value. The normality of the model residuals was assessed using the Shapiro-Wilk test. Continuous variables were summarised using number of observations (n), mean, standard deviation, median, minimum and maximum. Frequencies and percentages were reported for all categorical data. All analyses and tabulations were performed using SAS (version 9.3; SAS, Cary, NC, USA).

Ralinepag plasma concentrations were limited to pre-dose $\left(C_{\min }\right)$ and $4 \mathrm{~h}$ post-dose at designated study visits. Ralinepag plasma steady-state was determined by regressing $C_{\min }$ values over time and the resultant slope tested for its difference from zero.

\section{Results}

Patient disposition

61 patients were enrolled between January 2015 and June 2017 at 28 study centres in nine countries including the United States, Australia, Poland, Romania, Bulgaria, Serbia, Spain, Hungary and Czech Republic. Of the 61 patients, 52\% had idiopathic PAH, $8 \%$ had heritable $\mathrm{PAH}, 7 \%$ had drug- or toxin-induced PAH and $33 \%$ had associated PAH. Patients were predominantly female (86\%) with a mean age of 49.4 years and body weight of $73.2 \mathrm{~kg}$ at enrolment. The mean \pm sD baseline 6MWD was 378.4 $\pm 103.9 \mathrm{~m}$ and mean \pm SD baseline PVR was $717.4 \pm 414.8 \mathrm{dyn} \cdot \mathrm{s} \cdot \mathrm{cm}^{-5} .40$ patients were randomised to receive ralinepag and 21 to receive placebo (figure 2). Demographics and baseline characteristics of treatment groups are presented in table 1.

$34(85.0 \%)$ patients in the ralinepag group and 19 (90.5\%) in the placebo group completed the study. PVR was imputed for six ralinepag and two placebo patients for the primary end-point.

The overall mean \pm SD duration of treatment exposure including dosing interruptions was comparable between the ralinepag versus placebo groups; $168.70 \pm 46.5$ versus $180.1 \pm 13.8$ days, respectively. Ralinepag total daily maintenance doses were titrated as high as $600 \mu \mathrm{g}$, with the most common dose being $400 \mu \mathrm{g}$. 


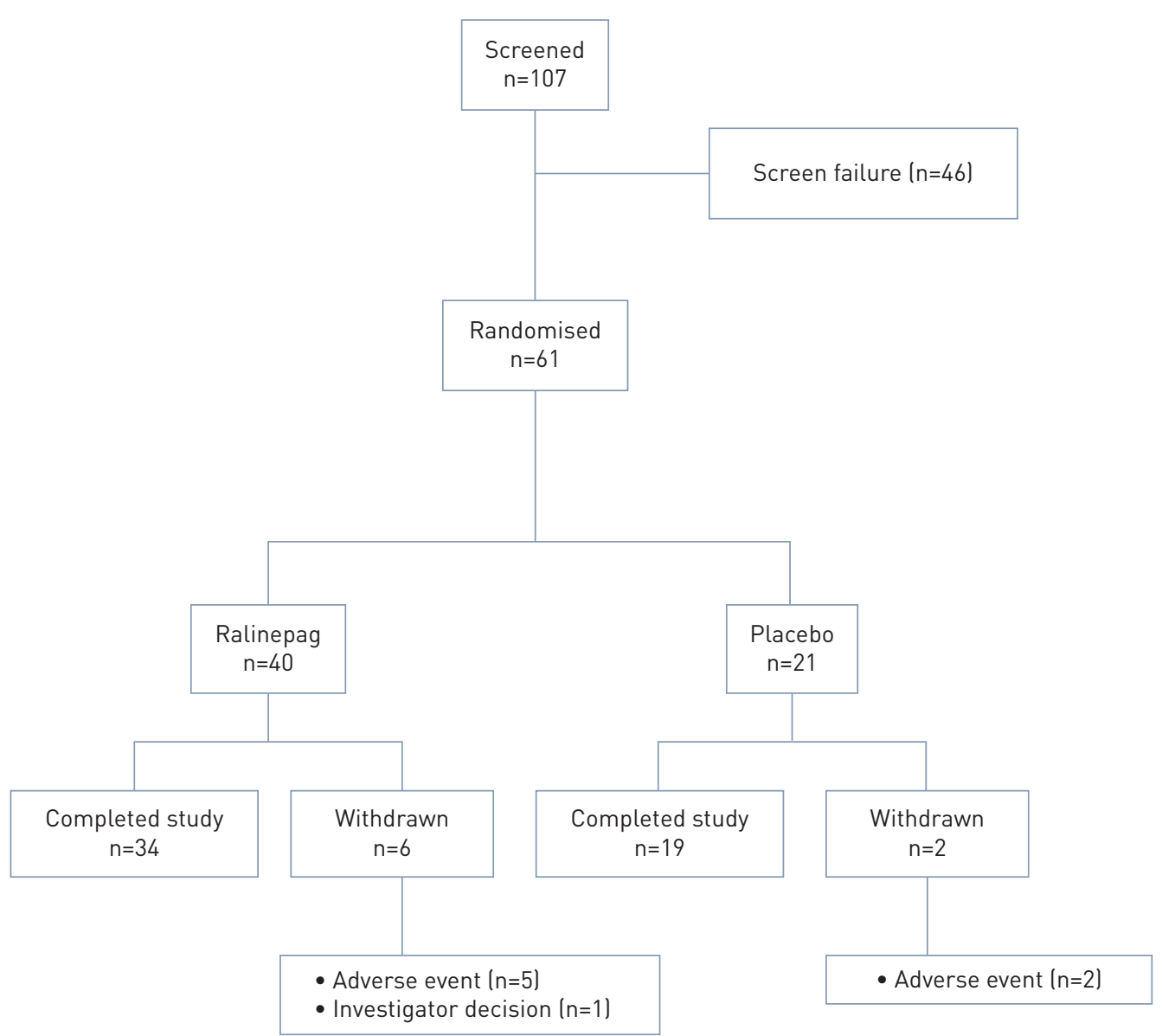

FIGURE 2 Patient disposition.

Efficacy

Following 22 weeks of treatment, a significant reduction in median PVR of $163.9 \mathrm{dyn} \cdot \mathrm{s} \cdot \mathrm{cm}^{-5}$ was observed in the ralinepag-treated patients, compared with a median increase of $0.7 \mathrm{dyn} \cdot \mathrm{s} \cdot \mathrm{cm}^{-5}$ observed with placebo ( $p=0.02$; figure 3 ), meeting the primary end-point of the study. These results, which imputed values for missing final data, were corroborated by the completers analysis and other sensitivity analyses, in which a median reduction in PVR of $169.7 \mathrm{dyn} \cdot \mathrm{s} \cdot \mathrm{cm}^{-5}$ for ralinepag and $3.1 \mathrm{dyn} \cdot \mathrm{s} \cdot \mathrm{cm}^{-5}$ for placebo were observed. In the completers analysis, a statistically significant reduction from baseline in PVR by $19.8 \%$ was observed in the ralinepag patients $(\mathrm{p}<0.0001)$.

There was a $29.8 \%$ reduction in PVR (least-squares mean) compared with placebo $(\mathrm{p}=0.03)$, which included a $20.1 \%$ decrease from baseline for ralinepag-treated subjects.

At week 22, changes from baseline in $6 \mathrm{MWD}$ with ralinepag were not significantly different than placebo, with least-squares mean 6MWD increases of $36.2 \mathrm{~m}$ for ralinepag treated patients and $29.4 \mathrm{~m}$ for placebo $(\mathrm{p}=0.90)$.

The proportion of patients with clinical worsening was lower with ralinepag versus placebo (2.5\% versus $9.5 \%)$, but the difference between the groups was not statistically significant $(\mathrm{p}=0.26)$. Notably, significantly fewer ralinepag- than placebo-treated patients deteriorated in WHO/NYHA functional class ( $2.5 \%$ versus $19.0 \% ; \mathrm{p}=0.03)$.

Exploratory analyses demonstrated that ralinepag treatment trended toward a reduction in mean NT-proBNP (-304.9 versus $\left.35.4 \mathrm{pg} \cdot \mathrm{mL}^{-1} ; \mathrm{p}=0.25\right)$ compared with placebo, but the treatment group differences were not statistically significant (figure 4).

Patients treated with ralinepag demonstrated significant reductions relative to placebo in mean pulmonary arterial pressure (PAP) (ralinepag $-6.1 \mathrm{mmHg}$, placebo $2.9 \mathrm{mmHg}$; $=0.028$ ), systemic vascular resistance (SVR) (ralinepag $-258.7 \mathrm{dyn} \cdot \mathrm{s} \cdot \mathrm{cm}^{-5}$, placebo $+152.9 \mathrm{dyn} \cdot \mathrm{s} \cdot \mathrm{cm}^{-5} ; \mathrm{p} \leqslant 0.001$ ) and arterial blood pressure (ralinepag $-8.2 \mathrm{mmHg}$, placebo $-2.7 \mathrm{mmHg}$; $=0.001$ ) (table 2). Cardiac index was higher with ralinepag compared with placebo, but the treatment effect was not statistically significant. 


\begin{tabular}{|c|c|c|c|c|}
\hline & All patients & Ralinepag & Placebo & p-value \\
\hline Subjects n & 61 & 40 & 21 & \\
\hline Age years $\#$ & $49.4(19-73)$ & $46.2(19-68)$ & $55.6(29-73)$ & $0.0057^{+}$ \\
\hline Female & $53(87)$ & 33 (83) & $20(95)$ & \\
\hline \multicolumn{5}{|l|}{ Ethnicity } \\
\hline Caucasian & 57 (93) & $38(95)$ & $19(91)$ & \\
\hline Other & 4 (7) & $2(5)$ & $2(10)$ & \\
\hline PVR dyn.s. $\mathrm{cm}^{-5}$ mean (median) & $717(576)$ & 780 (705) & $598(480)$ & $0.110^{+}$ \\
\hline 6MWD m mean (median) & $378(400)$ & 393 (405) & 351 (367) & \\
\hline \multicolumn{5}{|l|}{ WHO FC $\#$} \\
\hline II & $34(56)$ & $22(55)$ & $12(57)$ & $0.800^{\S}$ \\
\hline III & $26(43)$ & $17(43)$ & $9(43)$ & \\
\hline IV & $1(2)$ & 1 (3) & 0 & \\
\hline \multicolumn{5}{|l|}{ Aetiology of PAH } \\
\hline Idiopathic PAH & $21(52.5)$ & $11(52.4)$ & $32(52.5)$ & \\
\hline Heritable PAH & $4(10.0)$ & $1(4.8)$ & 5 (8.2) & \\
\hline Drugs or toxins & $4(10.0)$ & $0(0.0)$ & $4(6.6)$ & \\
\hline Associated $\mathrm{PAH}^{\Uparrow}$ & $11(27.5)$ & $9(42.9)$ & 20 (32.8) & \\
\hline NT-proBNP pg $\cdot \mathrm{mL}^{-1}$ mean (median) ${ }^{\#}$ & 980 (343) & 792 (335) & $1362(343)$ & $0.700^{+}$ \\
\hline \multicolumn{5}{|l|}{ Background PAH therapy \% } \\
\hline Monotherapy & 41 & 35 & 52 & \\
\hline Combination therapy & 59 & 65 & 48 & \\
\hline \multicolumn{5}{|l|}{ Monotherapy } \\
\hline ERA $^{\#}$ & $6(10)$ & $2(5)$ & $4(19)$ & $0.170^{f}$ \\
\hline PDE5i & $19(31)$ & $12(30)$ & 7 (33) & \\
\hline \multicolumn{5}{|l|}{ Combination therapy } \\
\hline ERA+PDE5i & $34(56)$ & $24(60)$ & $10(48)$ & $0.420^{f}$ \\
\hline $\mathrm{ERA}+\mathrm{sGCS}$ & $2(3)$ & $2(5)$ & $0(0)$ & \\
\hline New PAH treatment within 3-6 months of day 1 & $13(21)$ & $5(13)$ & $8(38)$ & $0.049^{f}$ \\
\hline \multicolumn{5}{|c|}{ 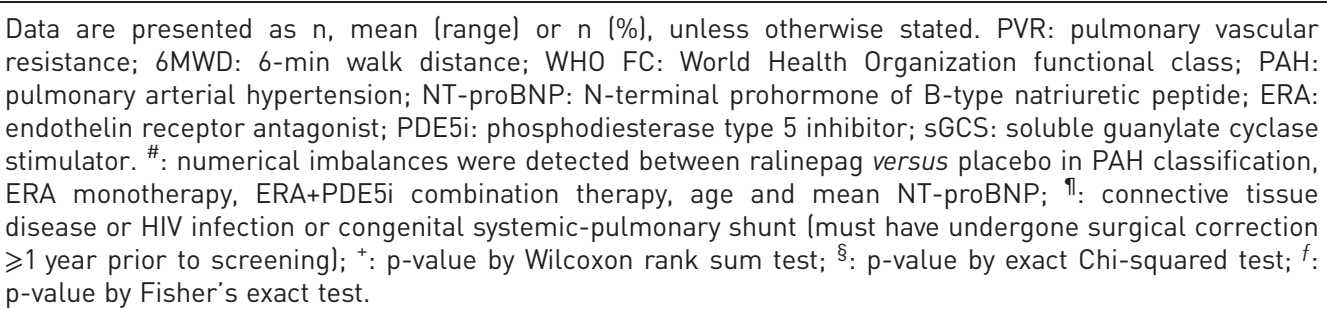 } \\
\hline
\end{tabular}

\section{Safety and adverse events}

Not unexpectedly, both SVR and systemic blood pressure decreased with ralinepag, but symptomatic hypotension was not observed. No other clinically meaningful changes in vital signs or laboratory tests from baseline to week 22 were observed. The most common reason for study withdrawal among both

FIGURE 3 Ralinepag significantly reduced pulmonary vascular resistance (PVR) compared with placebo. Within-group geometric mean ratio (GMR) percentage change: placebo $-0.4 \%$, ralinepag $-26.1 \%$. Intentionto-treat population $(n=61)$ imputation of missing values. Median change used due to non-normal distribution, mean absolute change from baseline: placebo $25.5 \mathrm{dyn} \cdot \mathrm{s} \cdot \mathrm{cm}^{-5}$, ralinepag $-175.7 \mathrm{dyn} \cdot \mathrm{s} \cdot \mathrm{cm}^{-5}$. The GMR of least-squares mean changes was $0.76,-25.8 \%$ change over compared placebo; $p=0.02$.

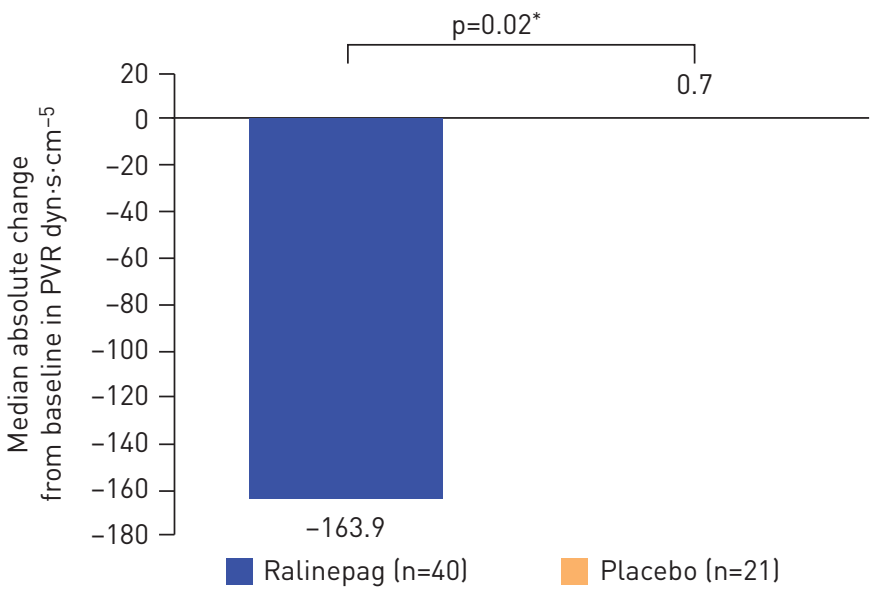




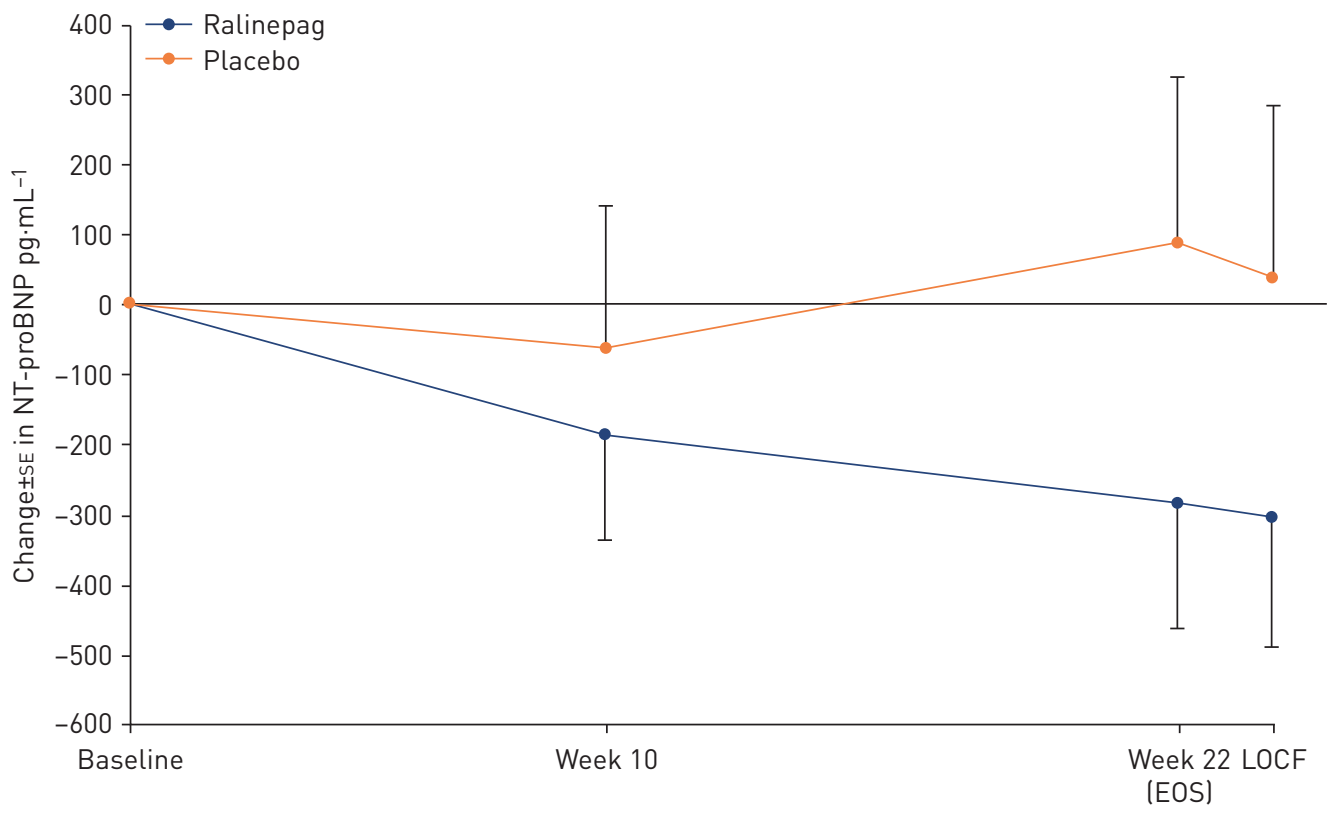

FIGURE 4 Change in N-terminal prohormone of B-type natriuretic peptide (NT-proBNP). EOS: end of study; LOCF: last observation carried forward.

groups was an adverse event. A summary of adverse events that occurred in $\geqslant 30 \%$ of patients is presented in table 3. All patients (100\%) in the ralinepag-treated group and $90.5 \%$ patients in the placebo group had at least one adverse event. Overall, $78.7 \%$ of adverse events were considered related to study drug, $95.0 \%$ in the ralinepag group and $47.6 \%$ in placebo. Of the adverse events related to study treatment, $20 \%$ of adverse events in the ralinepag group and $28.6 \%$ in placebo patients were mild in severity (asymptomatic or mild symptoms; clinical or diagnostic observations only; intervention not indicated) and $47.5 \%$ were considered moderate in severity (minimal, local or noninvasive intervention indicated) for ralinepag and $14.3 \%$ for placebo. Related events noted as severe (medically significant but not immediately life-threatening; hospitalisation or prolongation of hospitalisation indicated; disabling) occurred in $37.5 \%$ and $28.6 \%$ of ralinepag and placebo patients, respectively.

TABLE 2 Secondary haemodynamic parameters: change from baseline to week 22

\begin{tabular}{|c|c|c|c|c|c|}
\hline & \multicolumn{2}{|c|}{ Baseline } & \multicolumn{2}{|c|}{$\begin{array}{c}\text { Change from baseline to } \\
\text { week } 22^{\#}\end{array}$} & \multirow[t]{2}{*}{ p-value ${ }^{\text {? }}$} \\
\hline & Ralinepag & Placebo & Ralinepag & Placebo & \\
\hline Subjects $\mathrm{n}$ & 34 & 19 & 34 & 19 & \\
\hline $\begin{array}{l}\text { Mean pulmonary arterial } \\
\text { pressure } \mathrm{mmHg}\end{array}$ & $51.0 \pm 15.26$ & $43.1 \pm 10.08$ & $-6.1 \pm 1.5$ & $-2.9 \pm 2.0$ & 0.028 \\
\hline Cardiac index $\mathrm{L} \cdot \mathrm{min} \cdot \mathrm{m}^{-2}$ & $2.6 \pm 0.60$ & $2.9 \pm 0.49$ & $0.31 \pm 0.12$ & $-0.0 \pm 0.16$ & 0.183 \\
\hline $\begin{array}{l}\text { Mixed venous oxygen saturation } \\
\%\end{array}$ & $65.0 \pm 7.86$ & $68.0 \pm 6.28$ & $1.0 \pm 1.05$ & $0.6 \pm 1.56$ & 0.412 \\
\hline Right atrial pressure $\mathrm{mmHg}$ & $8.1 \pm 6.14$ & $8.7 \pm 5.06$ & $-0.3 \pm 0.68$ & $-2.0 \pm 0.89$ & 0.118 \\
\hline $\begin{array}{l}\text { Pulmonary capillary wedge } \\
\text { pressure } \mathrm{mmHg}\end{array}$ & $9.3 \pm 2.93$ & $10.5 \pm 3.17$ & $-0.4 \pm 0.59$ & $-0.3 \pm 0.77$ & 0.119 \\
\hline SVR dyn $\cdot S \cdot \mathrm{cm}^{-5}$ & $\begin{array}{r}1449.0 \\
\pm 378.53\end{array}$ & $\begin{array}{r}1309.9 \\
\pm 286.65\end{array}$ & $\begin{array}{l}-258.7 \\
\pm 286.54\end{array}$ & $\begin{array}{c}152.9 \\
\pm 374.47\end{array}$ & $<0.001$ \\
\hline $\begin{array}{l}\text { Mean arterial blood pressure } \\
\mathrm{mmHg}\end{array}$ & $89.6 \pm 16.13$ & $86.8 \pm 10.55$ & $-8.2 \pm 1.83$ & $-2.7 \pm 2.35$ & 0.001 \\
\hline Heart rate bpm & $77.1 \pm 13.61$ & $71.6 \pm 7.87$ & $0.9 \pm 1.92$ & $-0.8 \pm 2.49$ & 0.961 \\
\hline
\end{tabular}

Data are presented as mean \pm SD, unless otherwise stated. SVR: systemic vascular resistance; bpm: beats per minute. \#: least-squares mean \pm SEM change from baseline; ๆ: $p$-value based on a stratified Wilcoxon rank text: ANCOVA model with three factors (treatment, World Health Organization/New York Heart Association functional class, background pulmonary arterial hypertension therapy). 
TABLE 3 Adverse events occurring in $\geqslant 30 \%$ of patients in either the ralinepag or placebo groups during the 22-week study

\begin{tabular}{lcc} 
& Ralinepag & Placebo \\
\hline Subjects & 40 & 21 \\
Headache & $78(31)$ & $29(6)$ \\
Nausea & $50(20)$ & $24(5)$ \\
Diarrhoea & $48(19)$ & $14(3)$ \\
Pain in jaw & $35(14)$ & $14(3)$ \\
Flushing & $33(13)$ & $5(1)$ \\
\hline
\end{tabular}

Data are presented as $\mathrm{n}$ or $\%(\mathrm{n})$.

For patients treated with ralinepag, the most common adverse events reported during the study were headache $(n=31,78 \%)$, nausea $(n=20,50 \%)$ and diarrhoea $(n=19,48 \%)$.

When the adverse event data were analysed in 4-week periods (figure 5), a notable decline in adverse events was observed during the maintenance phase (weeks 10-22) relative to the dose titration phase (weeks 1-9). For example, the percentage of patients that experienced a headache was lower during weeks $10-14$ of the maintenance phase (44.4\%) relative to weeks $4-9$ of the dose titration phase (75\%) and continued to decline through the end of the study. Similar trends were observed for nausea and diarrhoea. For patients treated with placebo, the frequency of events was similar over the dose-titration period compared with the maintenance period.

Of the ralinepag-treated patients, five withdrew from the study due to adverse events determined to be at least possibly related to study drug by the investigator (myalgia, headache, nausea, pain of skin, eyelid oedema, exfoliative dermatitis, face oedema, decreased appetite and ECG QT prolongation). Fewer patients treated with ralinepag $(n=4,10 \%)$ experienced serious adverse events as compared to placebo $(n=6$, 28.6\%). Serious adverse events in the placebo and ralinepag-treated groups were deemed unrelated to study drug by the study investigator with the exception of ECG QT prolongation in one ralinepag patient. This patient had evidence of an intraventricular conduction disturbance (IVCD) at baseline and throughout the trial as evidenced by intermittent incomplete right bundle branch block. In patients with IVCD, the duration of repolarisation is increased, thereby confounding interpretation of the impact of exogenous factors (e.g. study drug exposure). Two ex-placebo subjects had fatal adverse events: one subject died from hypovolaemic shock ( $\sim 29$ days after the last dose of randomised study drug (placebo)) and one subject had myocardial infarction and cardiorespiratory arrest $(\sim 7$ days after the last dose of randomised study drug (placebo)).

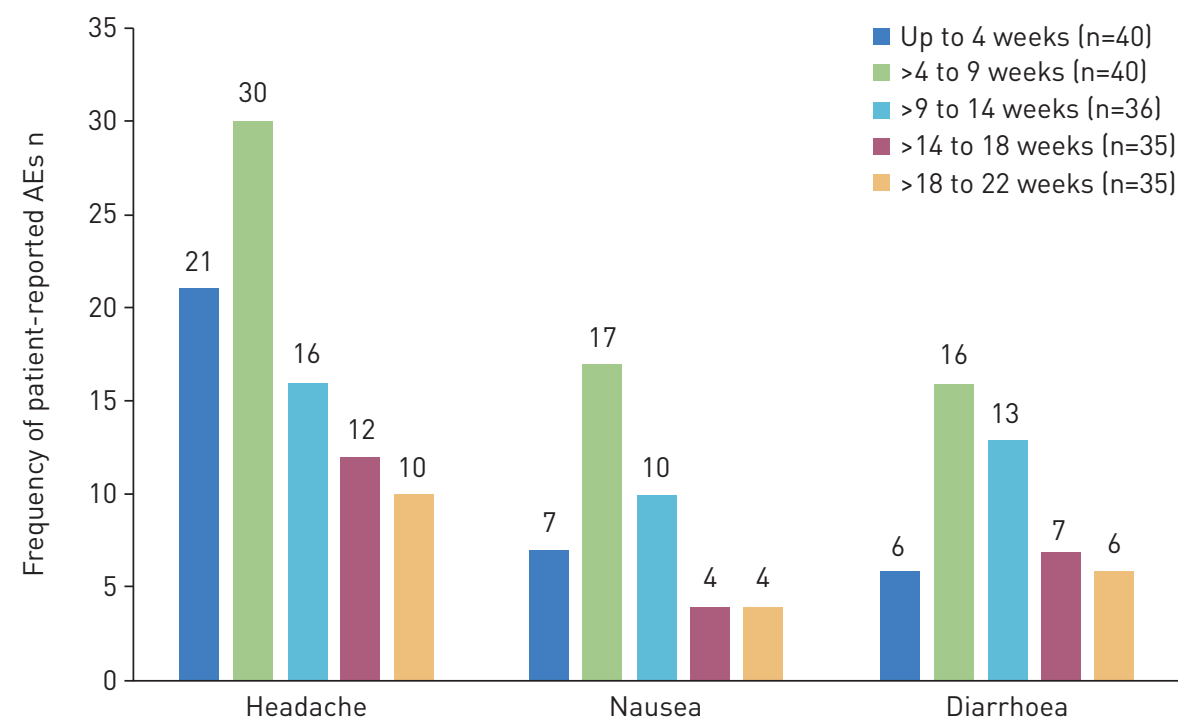

FIGURE 5 Frequency of patient-reported adverse events (AEs) as a function of time on ralinepag. 


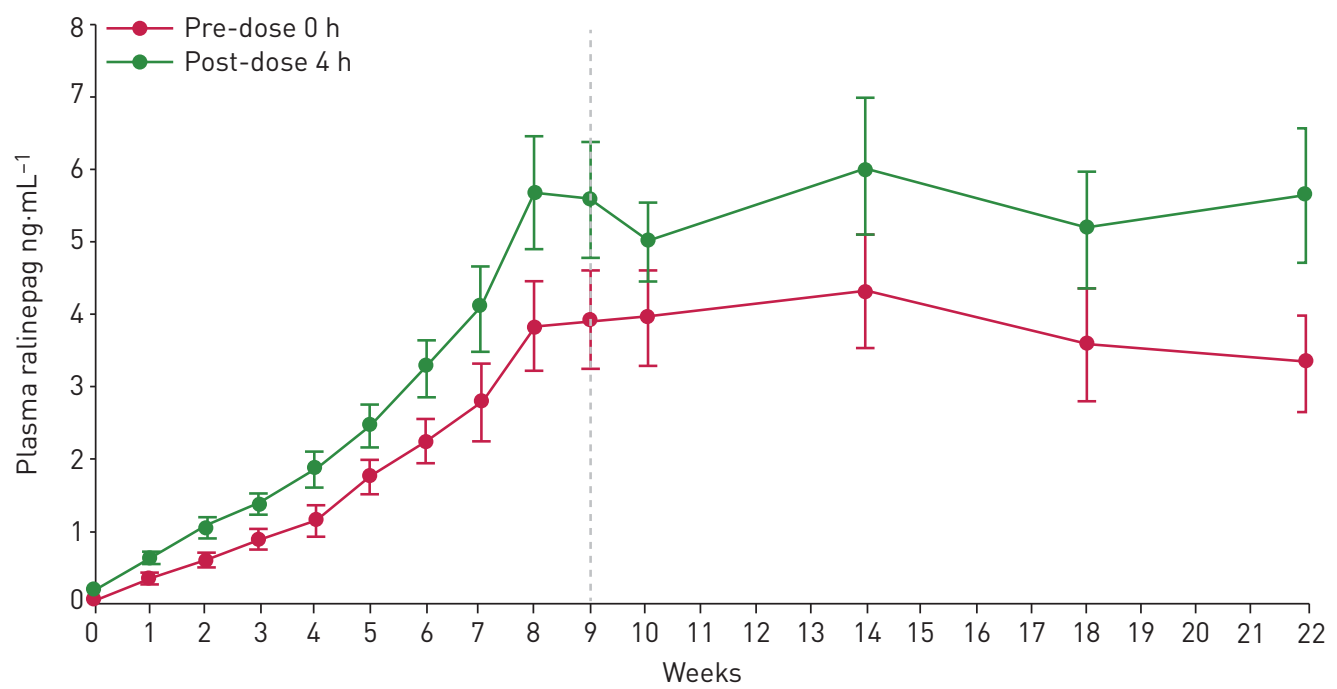

FIGURE 6 Mean士SEM plasma ralinepag steady-state plasma concentrations at pre-dose (trough) and $4 \mathrm{~h}$ post-dose during the 9-week dose titration and subsequent 13-week maintenance periods of the study (22-week total dosing duration).

\section{Pharmacokinetics}

Ralinepag immediate-release capsules were administered for the complete study. In patients receiving ralinepag, the mean \pm SD total daily dose at week 22 (end of maintenance period) in this study was 259 $\pm 168 \mu \mathrm{g}$. In general, mean ralinepag plasma concentrations at both evaluated time points increased during the dose-titration period and then reached approximate steady-state levels that were maintained throughout the maintenance period (figure 6). Overall, the mean steady-state week 22 plasma levels of ralinepag pre-dose and at $4 \mathrm{~h}$ post-dose were $3.23 \mathrm{ng} \cdot \mathrm{mL}^{-1}$ and $5.60 \mathrm{ng} \cdot \mathrm{mL}^{-1}$, respectively. The ralinepag plasma levels reflected by a mean total daily dose of $259 \mu \mathrm{g}$ appear similar (dose adjusted) to what was observed in the phase 1 study of healthy volunteers after twice-daily dosing. (SAD/MAD data on file).

\section{Discussion}

This study was designed to assess the efficacy, safety and tolerability of twice-daily ralinepag administered orally in a cohort of adult patients with PAH. Although some imbalances were observed between the treatment groups in several baseline measures, these are not surprising in light of the small sample size and 2:1 randomisation scheme. Imbalances were detected between ralinepag versus placebo in the mean duration of PAH, PAH classification, ERA monotherapy, ERA and PDE5 inhibitor combination therapy, age and mean NT-proBNP. While baseline PVR was higher in the ralinepag group, perhaps suggesting greater disease severity, baseline 6MWD was also higher and NT-ProBNP levels were lower in the ralinipag group; thus, no clear pattern of imbalance that might affect the interpretation of the results was identified. Similar findings were observed in the phase 2 trial of selexipag in PAH [8].

The study demonstrated a statistically significant absolute change in PVR, meeting the primary efficacy end-point. Expressed as percentage change relative to placebo, a $29.8 \%$ difference was observed, including a $20.1 \%$ decrease from baseline for the ralinepag-treated group. These reductions in PVR in the ralinepag group were observed despite patients already receiving either mono (35\%) or dual (65\%) combination PAH-specific background therapy. These findings are similar to those reported in the phase 2 study with selexipag [8], while oral treprostinil did not significantly reduce PVR in a single-centre, open-label study [9]. Additional signals of efficacy were also observed by assessing other haemodynamic parameters: ralinepag treatment led to greater mean reductions in both systolic and mean PAP when compared with placebo. While cardiac index and stroke volume index trended to increase with ralinepag compared with placebo, these treatment effects were not statistically significant; this result may have also been limited by the small sample size. Although SVR and blood pressure significantly decreased with ralinepag compared with placebo, there was no evidence of a significant compensatory increase in heart rate from baseline to week 22.

A statistically significant treatment effect in 6MWD was not observed $(+36.2 \mathrm{~m}$ ralinepag, $+29 \mathrm{~m}$ placebo; $\mathrm{p}=0.90$ ). This result was not unexpected, since the study was not powered to detect a difference in 6MWD. However, the $29.0 \mathrm{~m}$ increase in the placebo group was an unusual result. Similar phase 2 studies of imatinib [10], treprostinil [11] and selexipag [8] also failed to observe a statistically significant treatment 
effect in 6MWD relative to placebo. Notably, in each of these studies, the changes in 6MWD observed in the placebo group were relatively small $(-1.0 \mathrm{~m},+0.4 \mathrm{~m},+4.8 \mathrm{~m}$ for imatinib, selexipag and treprostinil, respectively). A post hoc analysis that examined differences in timing of new treatments prior to study start revealed significantly more patients in the placebo group relative to the ralinepag group had started a new PAH-specific background therapy within 3-6 months of study start (placebo $n=8,38 \%$; ralinepag $n=5$, $13 \% ; \mathrm{p}=0.045)$. In two previous studies utilising tadalafil [12] or riociguat [13], 6MWD continued to improve beyond week 12, suggesting the possibility that further improvement may be gained beyond 3 months after initiation of a new PAH-specific treatment

22 weeks of treatment with ralinepag was well tolerated by most patients in this study and treatment-related adverse events were generally consistent with the known safety profile of prostacyclin receptor agonists [14]. In order to optimise the benefit of treatment and prevent discontinuation due to intolerable adverse events, therapies targeting the prostacyclin pathway are initiated at low doses and up-titrated to a maximum tolerated maintenance dose [15]. In line with previous studies with prostacyclin analogues and IP receptor agonists, patients in the present study treated with ralinepag reported more frequent adverse events during the dose-titration phase from week 4 to week 9 , when compared with the maintenance period after week 9, indicating improvements in tolerability over time. The design of the 9-week dose titration scheme starts with low doses, which are increased weekly in small increments until a total daily dose of $200 \mu \mathrm{g}$, divided equally in two doses, is reached. The following week required a doubling of the dose to $400 \mu \mathrm{g}$, followed by an increase to $600 \mu \mathrm{g}$ in the final week. Dose-limiting adverse events triggered by aggressive titration may have caused patients to reach lower maximum tolerated doses than if a more gradual dose titration scheme was utilised.

Significantly fewer ralinepag- than placebo-treated patients deteriorated in WHO/NYHA functional class (one $(2.5 \%)$ out of 40 versus four $(19.0 \%)$ out of $21 ; \mathrm{p}=0.02)$. The WHO/NYHA functional class is one of the most powerful predictors of survival for $\mathrm{PAH}$, not only at diagnosis, but also during follow-up [15].

In the current study of ralinepag, NT-proBNP was chosen as an exploratory biomarker measure, since changes correlate with other predictors of survival in $\mathrm{PAH}$ patients [16, 17]. Although analyses demonstrated a greater mean reduction from baseline to week 22 with ralinepag versus placebo for NT-proBNP, the differences between treatment groups were not statistically significant $(\mathrm{p}=0.286$ and $\mathrm{p}=0.253$, respectively). Since this study was not powered to detect differences in this parameter, these results were not entirely unexpected.

Pharmacokinetic studies of ralinepag have demonstrated a long half-life of $\sim 24 \mathrm{~h}$, resulting in minimal fluctuation between peak and trough plasma concentrations at steady-state (SAD/MAD data on file). The plasma levels achieved in this study both pre-dose and $4 \mathrm{~h}$ post-dose $\left(3.23 \mathrm{ng} \cdot \mathrm{mL}^{-1}\right.$ and $5.60 \mathrm{ng} \cdot \mathrm{mL}^{-1}$, respectively) exceeded those that are associated with a pharmacological effect. The pharmacokinetic profile allows for twice-daily dosing of ralinepag, which may be advantageous to patients in terms of convenience and maintenance of the treatment effect throughout the dosing interval, and potentially minimising prostanoid-related adverse effects by reducing peaks in blood levels.

Our study has several limitations. The sample size was small and there were several imbalances at baseline between the two treatment arms. Additionally, the placebo group experienced a substantial increase in 6MWD, which is unusual and unexplained. Finally, while the PVR was significantly decreased with ralinepag (the primary end-point) as was PAP, changes in other parameters were not significantly changed. Accordingly, these results should be considered preliminary and interpreted with caution.

In conclusion, ralinepag, a next-generation, orally available, non-prostanoid selective prostacyclin receptor agonist significantly reduced PVR compared with placebo in patients with moderately symptomatic PAH. This effect was observed in subjects who were taking either monotherapy or combination therapy at the time of enrolment. Additional studies, including larger and more long-term clinical trials, are needed to confirm these findings.

Acknowledgements: The authors acknowledge the contribution of all patients and investigators who participated in this study. All authors read and approved the final version of the manuscript. Research was conducted at clinical study sites $(\mathrm{n}=28)$ in the USA, Australia, Poland, Romania, Bulgaria, Serbia, Spain, Hungary and Czech Republic.

Support statement: This study was sponsored by Arena Pharmaceuticals, Inc. Funding information for this article has been deposited with the Crossref Funder Registry.

Conflict of interest: F. Torres reports grants from UT Southwestern Medical Center, during the conduct of the study. H. Farber has nothing to disclose. A. Ristic has nothing to disclose. V. McLaughlin has nothing to disclose. J. Adams J. Zhang is an employee of Arena Pharmaceuticals. P. Klassen is an employee of Arena Pharmaceuticals. W. Shanahan is

a former employee of Arena Pharmaceuticals and hold shares. J. Grundy is an employee of Arena Pharmaceuticals.

I. Hoffmann is an employee of Arena Pharmaceuticals. C. Cabell is an employee of Arena Pharmaceuticals 
P. Escribano-Subias has nothing to disclose. N. Sood reports grants for research from The Ohio State University, during the conduct of the study. A. Keogh has nothing to disclose. G. D'Souza reports personal fees (consulting and travel) from Arena, outside the submitted work. L. Rubin has nothing to disclose.

\section{References}

1 Humbert M, Morrell NW, Archer SL, et al. Cellular and molecular pathobiology of pulmonary arterial hypertension. J Am Coll Cardiol 2004; 43: 12 Suppl. S, 13S-24S.

2 Budhiraja R, Tuder RM, Hassoun PM. Endothelial dysfunction in pulmonary hypertension. Circulation 2004; 109: $159-165$.

3 Midgett C, Stitham J, Martin K, et al. Prostacyclin receptor regulation - from transcription to trafficking. Curr Mol Med 2011; 11: 517-528.

4 Stitham J, Midgett C, Martin KA, et al. Prostacyclin: an inflammatory paradox. Front Pharmacol 2011; 2: 24.

5 McLaughlin VV, Shillington A, Rich S. Survival in primary pulmonary hypertension: the impact of epoprostenol therapy. Circulation 2002; 106: 1477-1482.

6 Clapp LH, Gurung R. The mechanistic basis of prostacyclin and its stable analogues in pulmonary arterial hypertension: role of membrane versus nuclear receptors. Prostaglandins Other Lipid Mediat 2015; 120: 56-71.

7 Tran TA, Kramer B, Shin YJ, et al. Discovery of 2-(((1r,4r)-4-((4-chlorophenyl)(phenyl)carbamoyl)oxy)methyl) cyclohexyl)methoxy)acetate (ralinepag): an orally active prostacyclin receptor agonist for the treatment of pulmonary arterial hypertension. J Med Chem 2017; 60: 913-927.

8 Simonneau G, Torbicki A, Hoeper MM, et al. Selexipag: an oral, selective prostacyclin receptor agonist for the treatment of pulmonary arterial hypertension. Eur Respir J 2012; 40: 874-880.

9 Chin KM, Ruggiero R, Bartolome S, et al. Long-term therapy with oral treprostinil in pulmonary arterial hypertension failed to lead to improvement in important physiologic measures: results from a single center. Pulm Circ 2015; 5: 513-520.

10 Ghofrani HA, Morrell NW, Hoeper MM, et al. Imatinib in pulmonary arterial hypertension patients with inadequate response to established therapy. Am J Respir Crit Care Med 2010; 182: 1171-1177.

11 Tapson VF, Jing ZC, Xu KF, et al. Oral treprostinil for the treatment of pulmonary arterial hypertension in patients receiving background endothelin receptor antagonist and phosphodiesterase type 5 inhibitor therapy (the FREEDOM-C2 study): a randomized controlled trial. Chest 2013; 144: 952-958.

12 Galiè N, Brundage BH, Ghofrani HA, et al. Tadalafil therapy for pulmonary arterial hypertension. Circulation 2009; 119: 2894-2903.

13 Ghofrani H-A, Hoeper MM, Halank M, et al. Riociguat for chronic thromboembolic pulmonary hypertension and pulmonary arterial hypertension: a phase II study. Eur Respir J 2010; 36: 792-799.

14 McLaughlin VV, Shah SJ, Souza R, et al. Management of pulmonary arterial hypertension. J Am Coll Cardio 2015; 65: 1976-1997.

15 Galiè N, Humbert M, Vachiery JL, et al. 2015 ESC/ERS Guidelines for the diagnosis and treatment of pulmonary hypertension: The Joint Task Force for the Diagnosis and Treatment of Pulmonary Hypertension of the European Society of Cardiology (ESC) and the European Respiratory Society (ERS): Endorsed by: Association for European Paediatric and Congenital Cardiology (AEPC), International Society for Heart and Lung Transplantation (ISHLT). Eur Respir J 2015; 46: 903-975.

16 Nagaya N, Nishikimi T, Uematsu M, et al. Plasma brain natriuretic peptide as a prognostic indicator in patients with primary pulmonary hypertension. Circulation 2000; 102: 865-870.

17 Fijalkowska A, Kurzyna M, Torbicki A, et al. Serum N-terminal brain natriuretic peptide as a prognostic parameter in patients with pulmonary hypertension. Chest 2006; 129: 1313-1321. 